\title{
Recent advances in food analysis
}

\author{
Jana Hajslova • Rudolf Krska • Michel W. F. Nielen
}

Published online: 22 May 2012

(C) Springer-Verlag 2012

Analytical and bioanalytical chemistry related to the food chain is a rapidly growing research area for many scientists in academia and industry. Major drivers behind this are, on the one hand, the development of high-quality "added-value" foods and, on the other hand, the recurrent food and feed incidents which, because of globalization, are not expected to decline. To deal with the increasing number of sample matrices and contaminants of interest, fast and accurate analytical methods are needed. Manufacturers of instruments and rapid screening assays have recognized this potential and nowadays specifically address this application area. This special issue on Recent Advances in Food Analysis provides the reader with a good overview of the current status and exciting developments in this field.

The analytical tools used in food analysis can be classified in a four-dimensional matrix of information content versus analysis time versus analyte bioactivity

Published in the special paper collection Recent Advances in Food Analysis with guest editors J. Hajslova, R. Krska, M. W. F. Nielen.

J. Hajslova

Department of Food Analysis and Nutrition,

Institute of Chemical Technology,

Technická 3,

16628 Prague 6, Czech Republic

R. Krska

Department of Natural Resources and Life Sciences,

Konrad-Lorenz-Str. 20,

3430 Tulln, Austria

M. W. F. Nielen $(\square)$

RIKILT-Institute of Food Safety,

Wagening University and Research Center,

P.O. Box 230, 6700 AE Wageningen, The Netherlands

e-mail: michel.nielen@wur.nl and analyte spatial distribution. In the area of rapid methods, for example dipsticks and immunological planar and bead suspension biosensing arrays, the trend is toward increased information content through multiplexing. In instrumental food analysis the trend is toward multi-analyte analysis - hundreds of pesticides and biotoxins in foods - by use of liquid chromatography mass spectrometry (LC-MS) operated in the multiple reaction monitoring or full-scan accurate mass mode, and still in a relatively short analysis time, because of recent developments in generic sample preparation and ultra-high performance separations using $<2 \mu \mathrm{m}$ LC stationary phase particles. The overwhelming amount of data from full-scan accurate-mass techniques requires urgent attention to the relevance of the data obtained. Currently two approaches can be distinguished, the development of sophisticated targeted and untargeted software tools for data reduction, alignment, searches, and multivariate comparison, and parallel bioactivity detection in which a bioactivity chromatogram pinpoints relevant areas in the reconstructed LC-MS chromatogram. So far, the spatial distribution of food components and contaminants has not been studied in much depth. However, ambient mass spectrometry techniques, for example direct analysis in real time (DART), are emerging and have the potential for future $2 \mathrm{D}$ surface imaging analysis, or even to yield $3 \mathrm{D}$ pictures after slicing of food products.

Nanoparticles are an entirely new challenge in food analysis. Apart from chemical identification and quantification, size distribution and surface-charge are also expected to be relevant. Maintaining sample integrity for nanoparticle traces during sample preparation from food matrices is an unprecedented challenge. 
We would like to express our sincere gratitude to the contributors - thanks to them, recent developments in all areas of food analysis can be presented in this special issue. We hope this collection of articles stimulates further research to better protect consumers by use of improved methods for sensitive and accurate determination of contaminants in foods which will also lead to improved exposure estimates and risk assessments. Last, but not least, we are also very grateful to the editorial team and the reviewers of Analytical and Bioanalytical Chemistry for their support and the fantastic cooperation.

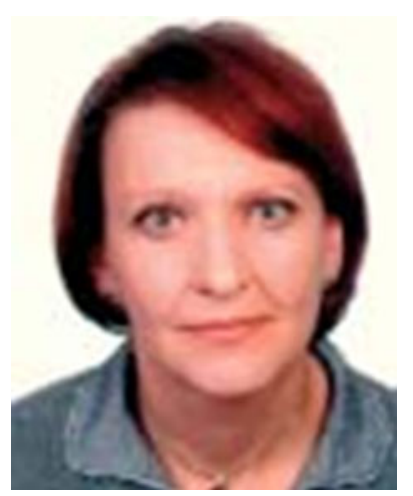

Jana Hajslova is a Professor at the Department of Food Chemistry and Analysis at the Institute of Chemical Technology in Prague (CZ). Her research interests focus on the fate of contaminants and toxicants in food chains and issues related to the implementation of novel strategies for their analysis. She is a board member of the EU-backed Advisory Group on Food Quality and Safety and a member of the European Union's Scientific Committee on Emerging and Newly Identified Health Risks. Professor Hajšlová has participated in many international research projects and has established close collaboration with several institutions, for example the UN's WHO and FAO, and the European Commission's Joint Research Centre. She has published widely on organic contaminants and food safety.

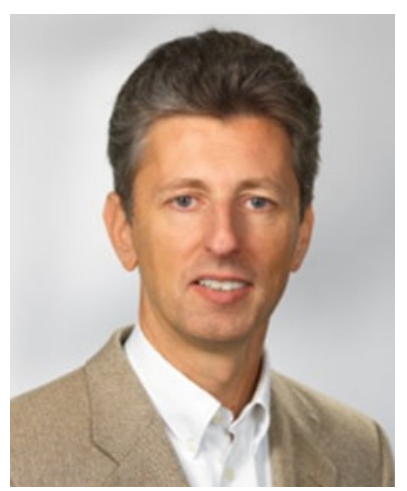

Rudolf Krska is Full Professor of Bioanalysis and Organic Trace Analysis and is head of the Department of Agrobiotechnology, IFA-Tulln, at the University of Natural Resources and Life Sciences, Vienna (BOKU). He obtained his degree in chemistry at the Vienna University of Technology and is an expert in food and feed analysis by chromatographic, mass spectrometric, and immunoanalytical techniques. As member of JECFA (Joint Expert Committee for Food Additives) of the FAO/WHO he has evaluated the effect of trichothecene mycotoxins on humans. He has received 6 scientific awards and is (co-)author of more than 258 scientific publications. In 2009/2010 he worked for a year as A/Chief of Health Canada's Food Research Division in Ottawa.

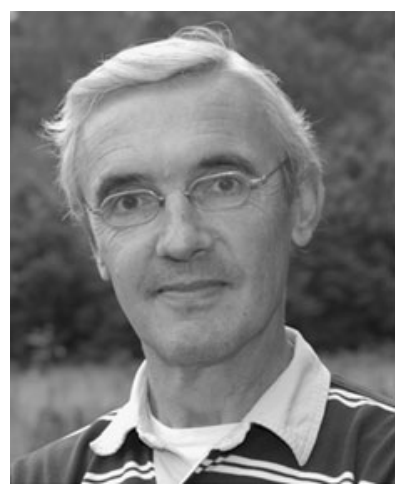

Michel W. F. Nielen is senior scientist in residue analysis at RIKILT and scientific director of TI-COAST, the Dutch public-private partnership on analytical science and technology. He holds a chair in detection of food contaminants at Wageningen University. He obtained his degree in analytical chemistry at the Free University of Amsterdam. He is co-founder and cochairman of the symposium series on recent advances in food analysis. He is (co-)author of more than 150 peer-reviewed publications. 